\title{
CHARACTERIZING THE COOL KEPLER OBJECTS OF INTERESTS. NEW EFFECTIVE TEMPERATURES, METALLICITIES, MASSES, AND RADII OF LOW-MASS KEPLER PLANET-CANDIDATE HOST STARS
}

\author{
Philip S. Muirhead ${ }^{1}$, Katherine Hamren $^{2}$, Everett Schlawin, Bárbara Rojas-Ayala ${ }^{3}$, \\ Kevin R. Cover ${ }^{4}$, And James P. Lloyd \\ Department of Astronomy, Cornell University, 122 Sciences Drive, Ithaca, NY 14853, USA; philm@ astro.caltech.edu \\ Received 2011 September 4; accepted 2012 April 5; published 2012 April 23
}

\begin{abstract}
We report stellar parameters for late-K and M-type planet-candidate host stars announced by the Kepler Mission. We obtained medium-resolution, $K$-band spectra of 84 cool $\left(T_{\text {eff }} \lesssim 4400 \mathrm{~K}\right)$ Kepler Objects of Interest (KOIs) from Borucki et al. We identified one object as a giant (KOI 977); for the remaining dwarfs, we measured effective temperatures $\left(T_{\text {eff }}\right)$ and metallicities $[\mathrm{M} / \mathrm{H}]$ using the $K$-band spectral indices of Rojas-Ayala et al. We determine the masses $\left(M_{\star}\right)$ and radii $\left(R_{\star}\right)$ of the cool KOIs by interpolation onto the Dartmouth evolutionary isochrones. The resultant stellar radii are significantly less than the values reported in the Kepler Input Catalog and, by construction, correlate better with $T_{\text {eff }}$. Applying the published KOI transit parameters to our stellar radius measurements, we report new physical radii for the planet candidates. Recalculating the equilibrium temperatures of the planet-candidates assuming Earth's albedo and re-radiation fraction, we find that three of the planet-candidates are terrestrial sized with orbital semimajor axes that lie within the habitable zones of their host stars (KOI 463.01, KOI 812.03, and KOI 854.01). The stellar parameters presented in this Letter serve as a resource for prioritization of future follow-up efforts to validate and characterize the cool KOI planet candidates.
\end{abstract}

Key words: planetary systems - stars: abundances - stars: fundamental parameters - stars: late-type - stars: low-mass

Online-only material: color figures, machine-readable table

\section{INTRODUCTION}

Estimating physical parameters of stars that host exoplanets is crucial for estimating the physical parameters of the exoplanets themselves. The wealth of detailed observations of the Sun has enabled precise calibration of stellar evolution models for Sunlike stars, such that the determination of fundamental stellar physical parameters (mass, effective temperature, luminosity, radius) from observed colors and spectra is routine and generally robust (e.g., Kurucz 1991; Nordström et al. 2004; Valenti \& Fischer 2005).

For cool dwarfs $\left(T_{\text {eff }} \lesssim 4400 \mathrm{~K}, M \lesssim 0.5 M_{\odot}\right)$, however, the situation is more complex. Low-mass stellar models are not as well calibrated, and their predictions differ substantially depending on assumptions such as the mixing length parameter. There are few $\mathrm{M}$ dwarfs that are bright enough and nearby enough for direct accurate parallax and radius measurements (e.g., Ségransan et al. 2003; Berger et al. 2006). Eclipsing binaries have been the primary source of radii for M dwarfs, but there is a discrepancy between observed radii and predictions from stellar evolution models (Ribas 2006; Torres 2011). The rapid rotation of these close binary systems may be responsible for the discrepancy; however, magnetohydrodynamic effects may suppress convection in their interiors, so these radii may not be representative of field objects (Chabrier et al. 2007; Kraus et al. 2011).

\footnotetext{
1 Current address: Department of Astronomy, California Institute of Technology, 1200 East California Boulevard, MC 249-17, Pasadena, CA 91125, USA.

2 Current address: Department of Astronomy and Astrophysics, University of California Santa Cruz, 1156 High Street, Santa Cruz, CA 95064, USA.

3 Current address: Department of Astrophysics, American Museum of Natural History, Central Park West at 79th Street, New York, NY 10024, USA.

4 Hubble Fellow.
}

Recently, M dwarfs have received increased attention in both transit and radial velocity searches for exoplanets (e.g., Charbonneau et al. 2009; Bean et al. 2010b; Johnson et al. 2010; Mahadevan et al. 2010; Muirhead et al. 2011; Bonfils et al. 2011), and exoplanet characterization efforts (e.g., Bean et al. 2010a; Désert et al. 2011; Croll et al. 2011), thanks to the higher detectability and characterization signals from orbiting low-mass exoplanets (Nutzman \& Charbonneau 2008). In 2011 February the Kepler Mission announced 997 objects whose light curves are consistent with the presence of transiting planets (Borucki et al. 2011), 74 of these Kepler Objects of Interest (KOIs) have $T_{\text {eff }}<4400 \mathrm{~K}$ in the Kepler Input Catalog (KIC; Batalha et al. 2010; Brown et al. 2011). A statistical analysis of the KOIs by Howard et al. (2011) reveals a substantial rise in the frequency of short-period, 2-4 $R_{\oplus}$ planets with decreasing $T_{\text {eff }}$ of their host stars, implying that the low-mass planet candidates detected around low-mass stars represent a ubiquitous population of planets in the Galaxy.

Stellar parameters in the KIC were inferred from a photometric survey of stars in the Kepler field of view. However, Brown et al. (2011) state that the KIC stellar parameters are reliable for Sun-like stars, but are "untrustworthy" for stars with $T_{\text {eff }}$ less than $3750 \mathrm{~K}$. The most reliable estimates of M dwarf masses and radii are derived by combining measured stellar luminosities with reliable mass-luminosity relations (e.g., Delfosse et al. 2000) and mass-radius relations predicted by stellar evolutionary models (e.g., Baraffe et al. 1998), often with corrections to account for discrepancies between measured and predicted radii (e.g., Torres 2007). Unfortunately, the low-mass KOIs do not have parallax measurements, which are necessary to estimate stellar luminosity and hence mass and radius with these methods.

Near-infrared spectroscopy offers a more robust method for determining physical parameters for low-mass stars that lack 
parallax measurements. The $K$ band $(2.0-2.4 \mu \mathrm{m})$ contains several useful spectral diagnostics including continuum regions sensitive to $T_{\text {eff }}$ (Covey et al. 2010) and absorption features that are sensitive to stellar metallicity (Rojas-Ayala et al. 2010, 2012), with minimal sensitivity to interstellar reddening.

In this Letter we report $T_{\text {eff }}$ and $[\mathrm{M} / \mathrm{H}]$ measurements of 84 low-mass KOIs using $K$-band spectroscopy. We interpolated $T_{\text {eff }}$ and $[\mathrm{M} / \mathrm{H}]$ onto the Dartmouth evolutionary isochrones (Dotter et al. 2008; Feiden et al. 2011), which reproduce measurements from optical long-baseline interferometry (OLBI) relatively well (see Figure 2) and contain a large spread of metallicity grid points, as required for reliable interpolation of stellar parameters. We report interpolated stellar masses and radii of the low-mass KOIs, and recalculate the planetary parameters based on the transit parameters in Borucki et al. (2011).

\section{OBSERVATIONAL CLASSIFICATION}

\subsection{Observations}

Observations were carried out with the TripleSpec Spectrograph at the Palomar Observatory 200 inch Hale Telescope. TripleSpec is a near-infrared slit-spectrograph covering $1.0-2.5 \mu \mathrm{m}$ simultaneously with a resolution of 2700 (Herter et al. 2008). Two positions on the slit, A and B, were used for each target, and exposures were taken in an ABBA pattern with $60 \mathrm{~s}$ integration times at each position. Multiple ABBA sets were taken and combined until each spectrum had a median per-channel signal to noise of at least 60 .

For telluric calibration we used SIMBAD to identify a grid of A0V stars in the Kepler field of view, and developed an observing sequence such that each KOI observation has a corresponding A0V star observation taken within 40 minutes and with an airmass difference less than 0.1 .

\subsection{Target Selection}

We observed all of the KOIs with KIC-ascribed effective temperatures less than $4400 \mathrm{~K}$ over seven nights in 2011 June. Of the 74 KOIs, 4 appeared to be double objects in the TripleSpec slit viewer with separations of less than 6 arcsec (roughly the size of the Kepler Spacecraft's point-spread function): KOI 326, KOI 641, KOI 249, and KOI 51. KOI 667 consisted of five objects within a 6 arcsec radius. These objects are not included in this survey.

In 2011 August we observed 15 additional KOIs with KICascribed $T_{\text {eff }}$ greater than $4400 \mathrm{~K}$, but with colors indicative of low-mass stars: $(J-K)>0.7,(r-i)>0.3$, or $(g-r)>1.0$. We include 13 of these KOIs in this Letter, as their spectra revealed $\mathrm{CO}$ features indicative of low-mass stars. In total, we obtained spectra of 82 KOIs.

\subsection{Data Reduction}

The spectra were extracted using a version of the Spextool program modified for the Palomar TripleSpec Spectrograph (Cushing et al. 2004; M. Cushing 2011, private communication). Spextool accepts data in ABBA format. The xtellcor package within Spextool accepts spectra of A0 stars and compares them to a model spectrum of Vega to identify and remove telluric absorption lines in a target spectrum (Vacca et al. 2003). Figure 1 plots three example spectra, with templates of similar spectra type and relevant spectral features indicated. The templates are taken from the IRTF Spectral Library (Cushing et al. 2005; Rayner et al. 2009).
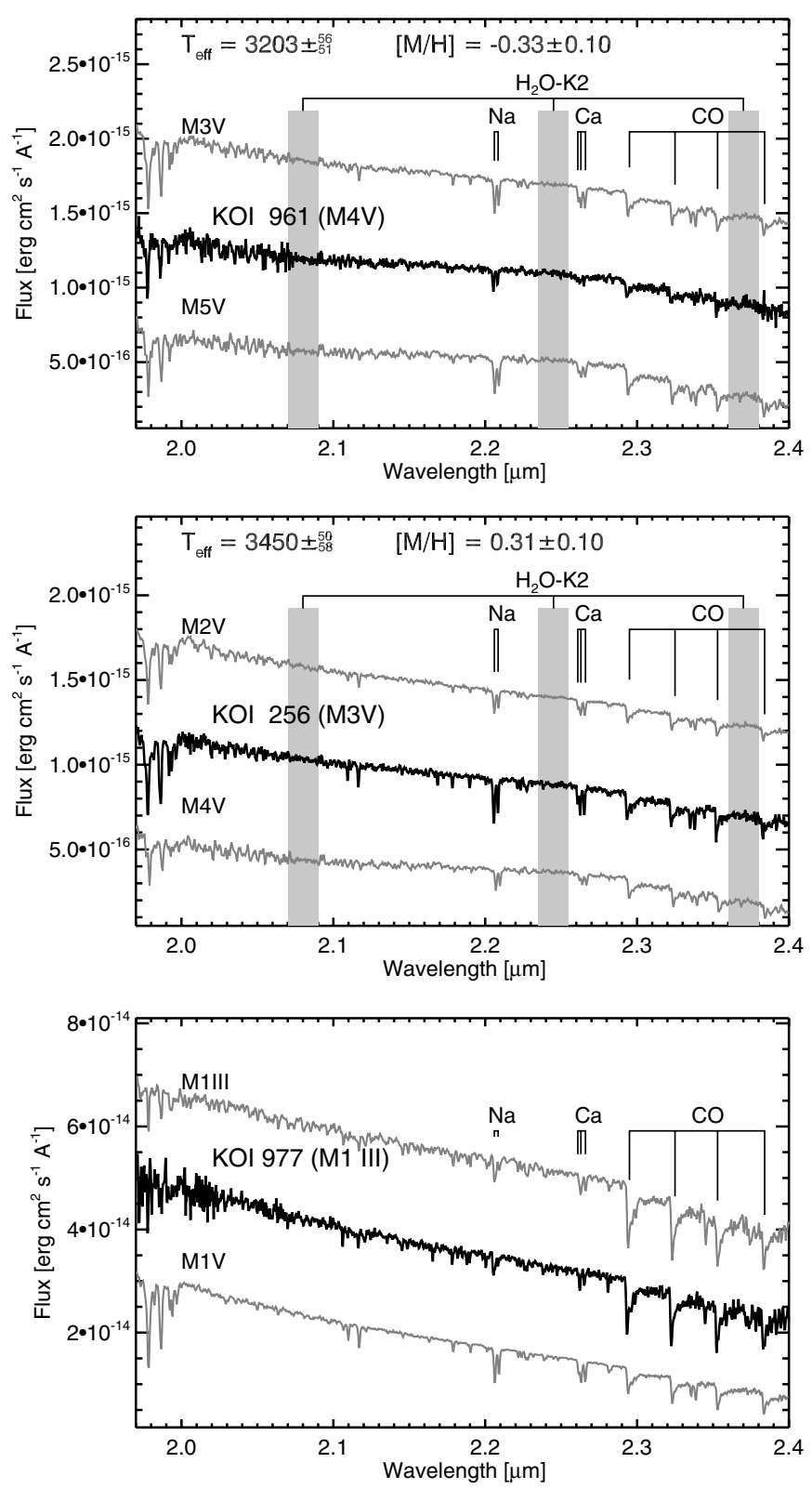

Figure 1. Palomar-TripleSpec $K$-band spectra of cool KOIs (black) with comparison KHM spectral type standards from the IRTF Spectral Library (gray; Cushing et al. 2005; Rayner et al. 2009). The templates are adjusted to the same scale as the KOI spectra using a ratio of the median flux in the $K$ band, and then artificially offset. We used the $\mathrm{H}_{2} \mathrm{O}-\mathrm{K} 2$ index to compute $T_{\text {eff }}$, which is calculated using regions dominated by water opacity, and we used the equivalent widths of the Na I doublet and Ca I triplet to measure $[\mathrm{M} / \mathrm{H}]$ (Rojas-Ayala et al. 2012). Top: KOI 961 is an example of a metal-poor star with little NaI and Ca I absorption (Muirhead et al. 2012). Middle: KOI 256 is an example of a metal-rich star with deep $\mathrm{Na}$ I and $\mathrm{Ca}$ I absorption. The metallicities $[\mathrm{M} / \mathrm{H}]$ are included, with uncertainties accounting for both random and systematic errors. Bottom: KOI 977 has a spectrum indicative of a giant with deep CO features but relatively weak $\mathrm{Na}$ I and $\mathrm{Ca}$ I absorption.

One star in our sample has a $K$-band spectrum consistent with a giant star, suggesting that the observed light curve is due to a stellar, rather than planetary, companion, or that the transit signal is due to an unresolved blend with an eclipsing binary. KOI 977 shows weak $\mathrm{Na}$ I and Ca I absorption and strong CO absorption, which qualitatively match IRTF template spectra of giant stars but not dwarf stars of the same spectral type. The spectrum is included in Figure 1, with a giant and dwarf template for comparison. 


\section{MEASUREMENT OF $T_{\text {eff }}$ AND [M/H]}

To measure $T_{\text {eff }}$ and $[\mathrm{M} / \mathrm{H}]$ of the remaining dwarfs, we measured three spectral indices from the $K$-band spectra: the equivalent widths of the $\mathrm{NaI}$ and $\mathrm{Ca}$ I lines, at 2.210 and $2.260 \mu \mathrm{m}$, respectively, and an index describing the change in flux between three $0.02 \mu \mathrm{m}$ wide bands dominated by water opacity-centered at $2.245,2.370,2.080 \mu \mathrm{m}$-called the $\mathrm{H}_{2} \mathrm{O}-\mathrm{K} 2$ index. Rojas-Ayala et al. (2012) describe the measurement of the $\mathrm{Na}$ I and $\mathrm{Ca}$ I equivalent widths, introduce the $\mathrm{H}_{2} \mathrm{O}-\mathrm{K} 2$ index, and derive relations between the spectral indices and $T_{\text {eff }}$, overall metallicity $([\mathrm{M} / \mathrm{H}])$, and KHM spectral type.

Briefly, we note that the Rojas-Ayala et al. (2012) $[\mathrm{M} / \mathrm{H}]$ relation was calibrated empirically using nearby $\mathrm{M}$ dwarfs with F-, G-, or K-type binary companions that have SPOCS $[\mathrm{M} / \mathrm{H}]$ measurements (Valenti \& Fischer 2005). Metallicity measurements for stars earlier than M0 $\left(T_{\text {eff }} \gtrsim 4000 \mathrm{~K}\right)$ represent an extrapolation of the $\mathrm{M}$ dwarf $[\mathrm{M} / \mathrm{H}]$ calibration. The $T_{\text {eff }}$ is calculated by interpolating the $[\mathrm{M} / \mathrm{H}]$ measurement and $\mathrm{H}_{2} \mathrm{O}-\mathrm{K} 2$ index onto a theoretical surface of $T_{\text {eff }}$ versus $[\mathrm{M} / \mathrm{H}]$ and $\mathrm{H}_{2} \mathrm{O}-\mathrm{K} 2$ index calculated to the BT-settl late-type model spectra of Allard et al. (2011). To validate the $T_{\text {eff }}$ measurement method, we compare $T_{\text {eff }}$ measurements by RojasAyala et al. (2012) to measurements from OLBI in Figure 3, panel (a).

The $T_{\text {eff }}$ surface is very metallicity insensitive $(<10 \mathrm{~K}$ offsets due to metallicity effects) for $3200<T_{\text {eff }}<3900$, and $[\mathrm{M} / \mathrm{H}]<0.3$. For higher temperatures the $\mathrm{H}_{2} \mathrm{O}-\mathrm{K} 2$ index saturates, where the saturation $T_{\text {eff }}$ depends on $[\mathrm{M} / \mathrm{H}]$. For stars with $\mathrm{H}_{2} \mathrm{O}-\mathrm{K} 2$ near the saturation, a slightly higher $\mathrm{H}_{2} \mathrm{O}-\mathrm{K} 2$ index converts to a large increase in the measured $T_{\text {eff. }}$. We accommodate this by providing asymmetric uncertainty estimates in $T_{\text {eff }}$ using a Monte Carlo approach described in Section 5. KOI 904 and KOI 956 had $\mathrm{H}_{2} \mathrm{O}-\mathrm{K} 2$ outside of the calculated surface, and are therefore not included in our results.

\section{DETERMINATION OF MASS AND RADIUS}

We place the low-mass KOIs on a grid of physical parameters based on the Dartmouth stellar evolution models (Dotter et al. 2008; Feiden et al. 2011). These models are in generally good agreement with OLBI observations (see Figure 2), but there may well be systematic offsets in mass, radius, or effective temperature, so these inferred physical parameters should be used with caution. We do not use the BCAH evolution models, as they are only available in two metallicities, $[\mathrm{M} / \mathrm{H}]=0.0$ and $[\mathrm{M} / \mathrm{H}]=-0.5$, and a comprehensive metallicity grid is required for reliable interpolation of our measurements.

All but one of the stars in our sample are treated homogeneously, so the relative radii, masses, and temperatures should be precise, even in the presence of model-dependent offsets. There is no model in the Dartmouth isochrones with the same metallicity and effective temperature as KOI 961, so this star is interpolated onto the $5 \mathrm{Gyr}$ BCAH isochrones (see Muirhead et al. 2012, however, for a more detailed analysis of this star). Stellar masses and radii are calculated by interpolation of the main sequence of a $5 \mathrm{Gyr}$ isochrone at the measured total metallicity and effective temperature, illustrated in Figure 4. The assumption of age does not significantly change the results. If very young ages are adopted, the masses and radii typically change only $0.1 \%$ between ages of 1 and $10 \mathrm{Gyr}$, and in all cases significantly less than the reported uncertainties.

We also apply our method to stars with $K$-band measurements in Rojas-Ayala et al. (2012) and radius measurements using

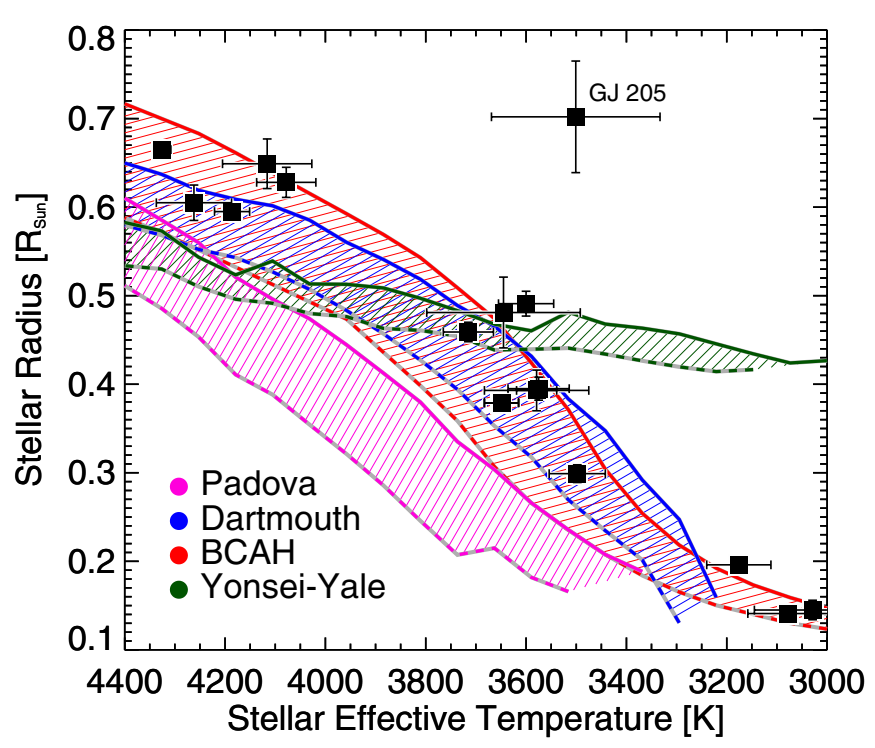

Figure 2. Predicted $R_{\star}$ vs. $T_{\text {eff }}$ for the $5 \mathrm{Gyr}$ isochrones of Padova (Girardi et al. 2002), Dartmouth (e.g., Dotter et al. 2008; Feiden et al. 2011), Yonsei-Yale (Yi et al. 2003), and BCAH (Baraffe et al. 1998), for metallicities between $[\mathrm{M} / \mathrm{H}]=-0.5$ (dashed lines) and $[\mathrm{M} / \mathrm{H}]=0.0$ (solid lines). We include empirical measurements of $R_{\star}$ and $T_{\text {eff }}$ for low-mass field stars using optical long-baseline interferometry (OLBI), with values taken from the literature (Berger et al. 2006; van Belle \& von Braun 2009; Boyajian et al. 2008; Lane et al. 2001; Demory et al. 2009; Ségransan et al. 2003; Kervella et al. 2008; von Braun et al. 2011). The Yonsei-Yale and Padova isochrones do not match the observations as well as the BCAH and Dartmouth isochrones. The BCAH isochrones are only available in two metallicities: $[\mathrm{M} / \mathrm{H}]=0.0$ and $[\mathrm{M} / \mathrm{H}]=$ -0.5 , necessitating poorly constrained interpolation and extrapolation if used to determine stellar mass and radius. Therefore, we chose to interpolate our $T_{\text {eff }}$ and $[\mathrm{M} / \mathrm{H}]$ measurements of the cool KOIs onto the Dartmouth isochrones. The OLBI measurements for GJ 205 (Ségransan et al. 2003) do not match any isochrone predictions, and this is likely the result of systematic errors in the OLBI measurements. We note that in the latest release of Kepler planet candidates, Batalha et al. (2012) confined the stellar parameters of the host stars to the Yonsei-Yale isochrones. This will produce systematically larger radii for the low-mass stars than is evidenced by long-baseline interferometry.

(A color version of this figure is available in the online journal.)

OLBI (see Figure 3, panels (b) and (c)). We find good agreement to within the estimated uncertainties.

\section{ERROR ANALYSIS}

We estimate the uncertainty in the equivalent width, $\mathrm{H}_{2} \mathrm{O}-\mathrm{K} 2$ index and $[\mathrm{M} / \mathrm{H}]$ measurements due to noise in the spectra using Monte Carlo simulations. Spextool reports errors for each channel of a reduced spectrum assuming photon noise and read noise in the target and telluric calibrator exposures. For each reduced spectrum, we created 1000 copies, each with random noise added to the spectral channels based on the error reported by Spextool. For each of the 1000 simulations, we measure the $\mathrm{Na}$ I and $\mathrm{Ca}$ I equivalent widths and $\mathrm{H}_{2} \mathrm{O}-\mathrm{K} 2$ index. We also calculate $[\mathrm{M} / \mathrm{H}]$ and $T_{\text {eff }}$, and interpolate those values onto the Dartmouth isochrones. The standard deviations of the quantities across the simulations are taken as the uncertainty in those quantities for a given KOI. All of the resulting distributions are reasonably symmetric, except for the $T_{\text {eff }}$ measurements, for which we report asymmetric uncertainties.

The $[\mathrm{M} / \mathrm{H}]$ and $T_{\text {eff }}$ measurements contain additional uncertainty from imperfections in the calibration relation. Possible sources of calibration errors include astrophysical scatter from non-perfect correlation between the indices and $[\mathrm{M} / \mathrm{H}]$, as well as errors in the relation coefficients due to noise in the calibration spectra. Rojas-Ayala et al. (2012) estimated the calibration 

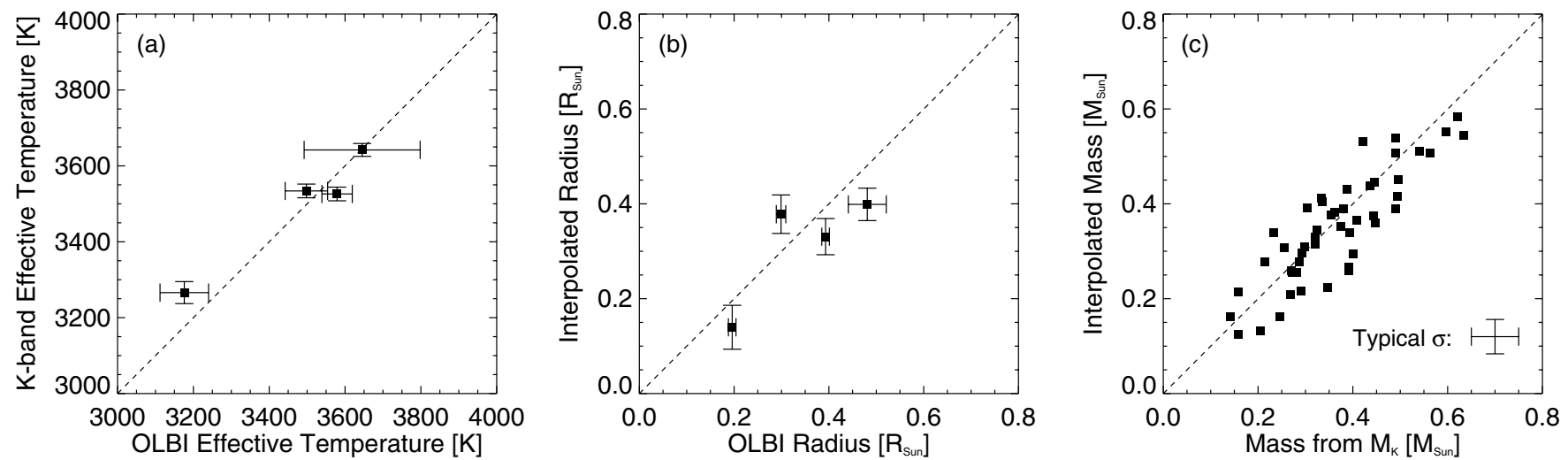

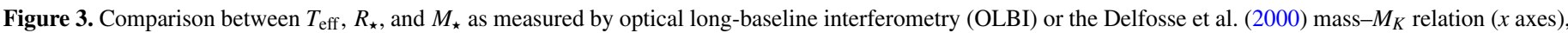

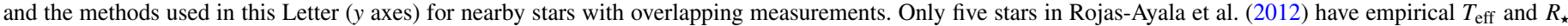

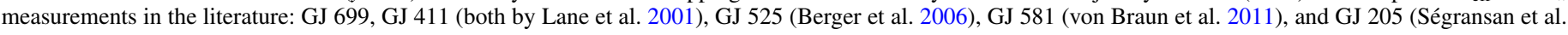

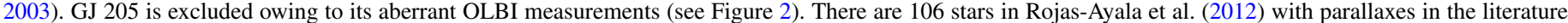

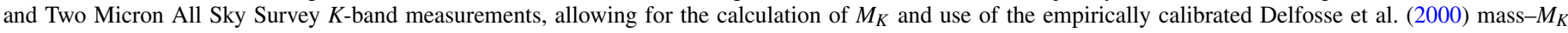

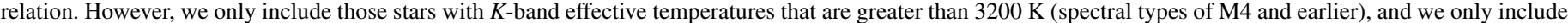

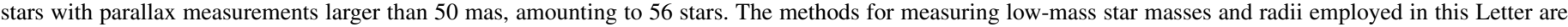
consistent with empirical measurements to within the calculated uncertainties.

errors contribute 0.1 dex of error to $[\mathrm{M} / \mathrm{H}]$ measurements, based on the root-mean-square residuals in the calibration fit. We estimate a $T_{\text {eff }}$ calibration uncertainty of $50 \mathrm{~K}$ by comparing the method to OLBI measurements (see Figure 3). We include these uncertainties into the $M_{\star}$ and $R_{\star}$ uncertainty estimates by adding additional Gaussian noise into the $[\mathrm{M} / \mathrm{H}]$ and $T_{\text {eff }}$ measurements of each Monte Carlo iteration with standard deviations of 0.1 and $50 \mathrm{~K}$, respectively.

A final uncertainty term arises from the inherent accuracy of the Dartmouth evolutionary isochrones. Figure 3 shows $R_{\star}$ as measured by optical long-baseline interferometry (OLBI) and $M_{\star}$ as measured using the Delfosse et al. (2000) mass- $M_{K}$ relation, versus measurements made using the Dartmouth interpolation method for nearby stars with $T_{\text {eff }}$ and $[\mathrm{M} / \mathrm{H}]$ measurements in Rojas-Ayala et al. (2012). We see no evidence for systematic offsets in the Dartmouth interpolations; nevertheless, we add in quadrature an additional uncertainty corresponding to $10 \%$ of the interpolated $M_{\star}$ and $R_{\star}$ to the Monte Carlo uncertainties based on the scatter in Figure 3.

\section{RESULTS AND DISCUSSION}

Table 1 lists the KOI planet candidates and our measurements of their host star parameters: spectral type, effective temperature, metallicity, mass, and radius, with corresponding uncertainty estimates. We also include our estimate of the planet-candidate radii, calculated by applying the $R_{P} / R_{\star}$ measurements reported in Borucki et al. (2011) to our measurements of $R_{\star}$. It should be noted that the appropriate limb-darkening coefficient could change as a result of the stellar classification, requiring a more sophisticated calculation of the new planet-candidate radii than in this Letter.

Figure 4 plots the effective temperatures, metallicities, masses, and radii from our analysis as well as the values in the KIC. Our stellar radii are systematically lower than the values reported in the KIC and, by construction, have better agreement with $T_{\text {eff }}$. The smaller stellar radii imply smaller planetcandidate radii, and many of the revised planet-candidate radii are smaller than 1 Earth radius.

The effective temperatures, radii, and masses of the KOIs imply different planet-candidate equilibrium temperature estimates, such that three planet candidates are terrestrial sized and

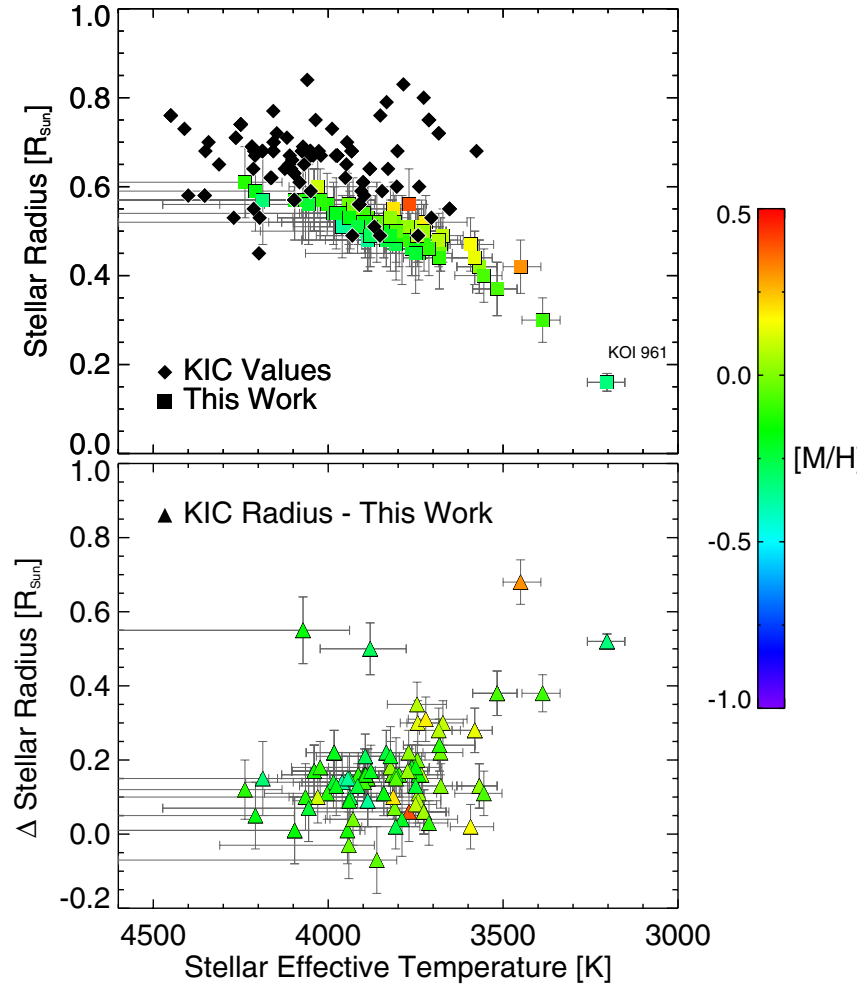

Figure 4. Temperature, metallicity, and radius determinations for the sample of low-mass KOIs. We use the $K$-band indices to measure $T_{\text {eff }}$ and $[\mathrm{M} / \mathrm{H}]$, then interpolate those values on the 5 Gyr Dartmouth isochrones to determine stellar mass and stellar radius (Dotter et al. 2008; Feiden et al. 2011). Top: stellar radius vs. $T_{\text {eff. }}$ Squares with error bars are the measurements in this Letter and black diamonds are the values from the KIC. Bottom: the difference between the radius determinations from the KIC and in this work, plotted against our $T_{\text {eff }}$ measurements. We dramatically revise the stellar radii of the coolest KOIs. KOI 961 is cooler than the available grid points in the Dartmouth isochrones, so we instead interpolate the $T_{\text {eff }}$ and $[\mathrm{M} / \mathrm{H}]$ onto the $5 \mathrm{Gyr}$ BCAH isochrones Baraffe et al. (1998). KOI 961 is the subject of the third paper in this series (Muirhead et al. 2012), wherein we use Barnard's star to more accurately estimate its stellar parameters and refit the transit parameters.

(A color version of this figure is available in the online journal.)

have equilibrium temperatures which may permit liquid water to reside on the planet surface, assuming Earth-like albedos and re-radiation fractions. We find that KOIs 463.01, 812.03, and 
Cool KOI Stellar Host and Planet-candidate Properties

\begin{tabular}{|c|c|c|c|c|c|c|c|c|c|c|}
\hline \multirow[b]{2}{*}{ KOI } & \multicolumn{2}{|c|}{$\begin{array}{l}\text { Borucki et al. (2011) } \\
\text { Transit Parameters }\end{array}$} & \multicolumn{3}{|c|}{$\begin{array}{c}K \text {-band Stellar Measurements } \\
\text { (This Work) }\end{array}$} & \multicolumn{2}{|c|}{$\begin{array}{c}\text { Dartmouth Stellar Interpolants } \\
\text { (This Work) }\end{array}$} & \multicolumn{3}{|c|}{$\begin{array}{l}\text { New Planet Parameters } \\
\text { (This Work) }\end{array}$} \\
\hline & $\begin{array}{c}P \\
\text { (days) }\end{array}$ & $R_{\mathrm{P}} / R_{\star}$ & $\begin{array}{l}T_{\text {eff }} \\
(\mathrm{K})\end{array}$ & {$[\mathrm{M} / \mathrm{H}]^{\mathrm{a}}$} & Sp. Type & $\begin{array}{c}M_{\star} \\
\left(M_{\odot}\right)\end{array}$ & $\begin{array}{c}R_{\star} \\
\left(R_{\odot}\right)\end{array}$ & $\begin{array}{c}a \\
(\mathrm{AU})\end{array}$ & $\begin{array}{c}R_{\mathrm{P}} \\
\left(R_{\oplus}\right)\end{array}$ & $\begin{array}{l}T_{\mathrm{eq}, \mathrm{P}} \\
(\mathrm{K})^{\mathrm{b}}\end{array}$ \\
\hline 104.01 & $2.5080910 \pm 1.3 \mathrm{e}-05$ & $0.035 \pm 4.8 \mathrm{e}-03$ & $4238_{-106}^{+459}$ & $-0.12 \pm 0.10$ & M0V & $0.64 \pm 0.08$ & $0.61 \pm 0.08$ & $0.03106 \pm 1.29 \mathrm{e}-03$ & $2.32 \pm 0.43$ & 827 \\
\hline 156.01 & $8.0414400 \pm 1.3 \mathrm{e}-04$ & $0.023 \pm 1.3 \mathrm{e}-02$ & $3983_{-81}^{+80}$ & $-0.20 \pm 0.10$ & M0V & $0.56 \pm 0.06$ & $0.54 \pm 0.06$ & $0.06482 \pm 2.35 \mathrm{e}-03$ & $1.36 \pm 0.78$ & 508 \\
\hline 156.02 & $5.1885600 \pm 1.2 \mathrm{e}-04$ & $0.020 \pm 1.4 \mathrm{e}-02$ & $\ldots$ & $\ldots$ & $\ldots$ & $\ldots$ & $\ldots$ & $0.04840 \pm 1.75 \mathrm{e}-03$ & $1.18 \pm 0.84$ & 588 \\
\hline 156.03 & $11.7761793 \pm 5.4 \mathrm{e}-05$ & $0.033 \pm 2.8 \mathrm{e}-04$ & & $\ldots$ & $\ldots$ & $\ldots$ & $\ldots$ & $0.08358 \pm 3.03 \mathrm{e}-03$ & $1.95 \pm 0.22$ & 447 \\
\hline 222.01 & $6.3123822 \pm 5.8 \mathrm{e}-05$ & $0.033 \pm 3.9 \mathrm{e}-04$ & $4096_{-187}^{+538}$ & $-0.17 \pm 0.11$ & MoV & $0.59 \pm 0.09$ & $0.57 \pm 0.09$ & $0.05611 \pm 2.81 \mathrm{e}-03$ & $2.05 \pm 0.31$ & 576 \\
\hline 222.02 & $12.7939701 \pm 3.0 \mathrm{e}-04$ & $0.026 \pm 6.5 \mathrm{e}-04$ & & & $\ldots$ & & & $0.08987 \pm 4.50 \mathrm{e}-03$ & $1.62 \pm 0.25$ & 455 \\
\hline 227.01 & $17.6607609 \pm 2.5 \mathrm{e}-04$ & $0.040 \pm 3.9 \mathrm{e}-04$ & $3745_{-59}^{+51}$ & $-0.02 \pm 0.10$ & M1V & $0.49 \pm 0.06$ & $0.47 \pm 0.05$ & $0.10496 \pm 3.91 \mathrm{e}-03$ & $2.06 \pm 0.24$ & 350 \\
\hline 247.01 & $13.8152399 \pm 3.2 \mathrm{e}-04$ & $0.031 \pm 1.1 \mathrm{e}-03$ & $3741_{-51}^{+54}$ & $0.03 \pm 0.10$ & M1V & $0.51 \pm 0.06$ & $0.49 \pm 0.06$ & $0.08992 \pm 3.29 \mathrm{e}-03$ & $1.64 \pm 0.20$ & 383 \\
\hline 248.01 & $7.2034941 \pm 6.5 \mathrm{e}-05$ & $0.039 \pm 5.5 \mathrm{e}-04$ & $3816_{-54}^{+141}$ & $-0.05 \pm 0.11$ & M1V & $0.54 \pm 0.06$ & $0.51 \pm 0.06$ & $0.05929 \pm 2.27 \mathrm{e}-03$ & $2.18 \pm 0.27$ & 495 \\
\hline 248.02 & $10.9140100 \pm 1.8 \mathrm{e}-04$ & $0.034 \pm 1.1 \mathrm{e}-03$ & $\ldots$ & $\ldots$ & $\ldots$ & $\ldots$ & $\ldots$ & $0.07821 \pm 3.00 \mathrm{e}-03$ & $1.90 \pm 0.24$ & 431 \\
\hline 248.03 & $2.5765359 \pm 3.3 \mathrm{e}-05$ & $0.027 \pm 1.2 \mathrm{e}-02$ & $\ldots$ & $\ldots$ & $\ldots$ & $\ldots$ & $\ldots$ & $0.02987 \pm 1.14 \mathrm{e}-03$ & $1.51 \pm 0.70$ & 697 \\
\hline
\end{tabular}

Notes.

${ }^{a}$ Metallicity measurements for stars earlier than M0 $\left(T_{\text {eff }} \lesssim 4000 \mathrm{~K}\right)$ represent an extrapolation of the Rojas-Ayala et al. (2012) M dwarf [M/H] calibration.

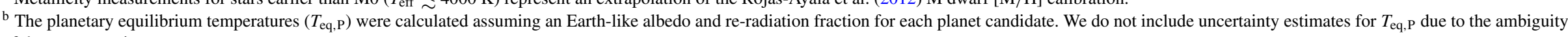
of those assumptions.

(This table is available in its entirety in a machine-readable form in the online journal. A portion is shown here for guidance regarding its form and content.) 
854.01 are less than $2 R_{\oplus}$ in size with equilibrium temperatures between $215 \mathrm{~K}$ and $275 \mathrm{~K}$ : rough limits to the habitable zone as calculated by Kasting et al. (1993). Whether liquid water can persist on the surfaces of terrestrial planets orbiting lowmass stars depends strongly on the individual evolution and atmospheric peculiarities of each system, but these KOIs are nevertheless compelling targets for future follow-up work.

A recent paper by Gaidos et al. (2012) compares the statistics from the M2K M-dwarf Doppler survey (e.g., Apps et al. 2010) with the Kepler results. They found inconsistencies which could be explained if many of the KOI stellar radii were underestimated, a result which is contradictory to our findings. However, the KIC $T_{\text {eff }}$ used to compare the M2K and Kepler samples differ from our measurements, and the two samples may have different metallicity distributions.

We thank Michael Cushing for providing us with a version of the Spextool package for the TripleSpec Spectrograph at Palomar. We also thank John Johnson for his thoughtful comments on the Letter. The Palomar 200 inch Telescope time was provided by Cornell University. K.R.C. acknowledges support for this work from the Hubble Fellowship Program, provided by NASA through Hubble Fellowship grant HST-HF-51253.01-A awarded by the STScI, which is operated by the AURA, Inc., for NASA, under contract NAS 5-26555.

\section{REFERENCES}

Allard, F., Homeier, D., \& Freytag, B. 2011, arXiv:1112.3591 Apps, K., Clubb, K. I., Fischer, D. A., et al. 2010, PASP, 122, 156 Baraffe, I., Chabrier, G., Allard, F., \& Hauschildt, P. H. 1998, A\&A, 337, 403

Batalha, N. M., Borucki, W. J., Koch, D. G., et al. 2010, ApJ, 713, L109

Batalha, N. M., Rowe, J. F., Bryson, S. T., et al. 2012, arXiv:1202.5852

Bean, J. L., Miller-Ricci Kempton, E., \& Homeier, D. 2010a, Nature, 468, 669

Bean, J. L., Seifahrt, A., Hartman, H., et al. 2010b, ApJ, 713, 410

Berger, D. H., Gies, D. R., McAlister, H. A., et al. 2006, ApJ, 644, 475

Bonfils, X., Delfosse, X., Udry, S., et al. 2011, arXiv:1111.5019

Borucki, W. J., Koch, D. G., Basri, G., et al. 2011, ApJ, 728, 117

Boyajian, T. S., McAlister, H. A., Baines, E. K., et al. 2008, ApJ, 683, 424
Brown, T. M., Latham, D. W., Everett, M. E., \& Esquerdo, G. A. 2011, AJ, 142, 112

Chabrier, G., Gallardo, J., \& Baraffe, I. 2007, A\&A, 472, L17

Charbonneau, D., Berta, Z. K., Irwin, J., et al. 2009, Nature, 462, 891

Covey, K. R., Lada, C. J., Román-Zúñiga, C., et al. 2010, ApJ, 722, 971

Croll, B., Albert, L., Jayawardhana, R., et al. 2011, ApJ, 736, 78

Cushing, M. C., Rayner, J. T., \& Vacca, W. D. 2005, ApJ, 623, 1115

Cushing, M. C., Vacca, W. D., \& Rayner, J. T. 2004, PASP, 116, 362

Delfosse, X., Forveille, T., Ségransan, D., et al. 2000, A\&A, 364, 217

Demory, B.-O., Ségransan, D., Forveille, T., et al. 2009, A\&A, 505, 205

Désert, J.-M., Bean, J., Miller-Ricci Kempton, E., et al. 2011, ApJ, 731, L40

Dotter, A., Chaboyer, B., Jevremović, D., et al. 2008, ApJS, 178, 89

Feiden, G. A., Chaboyer, B., \& Dotter, A. 2011, ApJ, 740, L25

Gaidos, E., Fischer, D. A., Mann, A. W., \& Lépine, S. 2012, ApJ, 746, 36

Girardi, L., Bertelli, G., Bressan, A., et al. 2002, A\&A, 391, 195

Herter, T. L., Henderson, C. P., Wilson, J. C., et al. 2008, Proc. SPIE, 7014, 70140X

Howard, A. W., Marcy, G. W., Bryson, S. T., et al. 2011, arXiv:1103.2541

Johnson, J. A., Aller, K. M., Howard, A. W., \& Crepp, J. R. 2010, PASP, 122, 905

Kasting, J. F., Whitmire, D. P., \& Reynolds, R. T. 1993, Icarus, 101, 108

Kervella, P., Mérand, A., Pichon, B., et al. 2008, A\&A, 488, 667

Kraus, A. L., Tucker, R. A., Thompson, M. I., Craine, E. R., \& Hillenbrand, L. A. 2011, ApJ, 728, 48

Kurucz, R. L. 1991, in Solar Interior and Atmosphere, ed. M. S. Matthews, A. N. Cox, \& W. C. Livingston (Tucson, AZ: Univ. Arizona Press), 663

Lane, B. F., Boden, A. F., \& Kulkarni, S. R. 2001, ApJ, 551, L81

Mahadevan, S., Ramsey, L., Wright, J., et al. 2010, Proc., SPIE, 7735

Muirhead, P. S., Edelstein, J., Erskine, D. J., et al. 2011, PASP, 123, 709

Muirhead, P. S., Johnson, J. A., Apps, K., et al. 2012, ApJ, 747, 144

Nordström, B., Mayor, M., Andersen, J., et al. 2004, A\&A, 418, 989

Nutzman, P., \& Charbonneau, D. 2008, PASP, 120, 317

Rayner, J. T., Cushing, M. C., \& Vacca, W. D. 2009, ApJS, 185, 289

Ribas, I. 2006, Ap\&SS, 304, 89

Rojas-Ayala, B., Covey, K. R., Muirhead, P. S., \& Lloyd, J. P. 2010, ApJ, 720, L113

Rojas-Ayala, B., Covey, K. R., Muirhead, P. S., \& Lloyd, J. P. 2012, ApJ, 748, 93

Ségransan, D., Kervella, P., Forveille, T., \& Queloz, D. 2003, A\&A, 397, L5

Torres, G. 2007, ApJ, 671, L65

Torres, G. 2011, arXiv: 1107.3088

Vacca, W. D., Cushing, M. C., \& Rayner, J. T. 2003, PASP, 115, 389

Valenti, J. A., \& Fischer, D. A. 2005, ApJS, 159, 141

van Belle, G. T., \& von Braun, K. 2009, ApJ, 694, 1085

von Braun, K., Boyajian, T. S., Kane, S. R., et al. 2011, ApJ, 729, L26

Yi, S. K., Kim, Y.-C., \& Demarque, P. 2003, ApJS, 144, 259 